\title{
Midbandgap electro-optic detection of Bloch oscillations
}

\author{
M. Först, ${ }^{*}$ G. Segschneider, T. Dekorsy, and H. Kurz \\ Institut für Halbleitertechnik II, Rheinisch-Westfälische Technische Hochschule Aachen, D-52056 Aachen, Germany \\ K. Köhler \\ Fraunhofer-Institut für Angewandte Festkörperphysik, D-79108 Freiburg, Germany
}

\begin{abstract}
Bloch oscillations excited in a biased $\mathrm{GaAs} / \mathrm{Al}_{x} \mathrm{Ga}_{1-x} \mathrm{As}$ superlattice are investigated in a time-resolved two-color electro-optic detection scheme. The detection of resonantly excited Bloch oscillations is based on the Pockels effect probed at a wavelength in the center of the band gap. The observed birefringence is induced by the macroscopic polarization of the electronic wave packets relative to the localized holes. The off-resonant detection away from optical transitions directly monitors the spatial dynamics of the electrons in amplitude and phase. The dependence of the amplitudes of the Bloch oscillating electrons on the applied electric fields are in good agreement with the electron-hole dipole lengths calculated by a quantum-mechanical model.
\end{abstract}

Bloch $^{1}$ and Zener ${ }^{2}$ have proposed in their theoretical studies on the dynamics of electronic wave packets in periodic potentials a temporal and spatial oscillation of the carriers in the presence of a static electric field. The frequency $\nu$ and the spatial amplitude $L$ of these Bloch oscillations (BO's) are

$$
\nu=e F d / h \text { and } L=\Delta / e F
$$

respectively, where $F$ is the applied electric field, $d$ the potential period, $h$ Planck's constant, and $\Delta$ the electronic bandwidth. As proposed by Esaki and Tsu, this intriguing phenomenon can be realized in a biased semiconductor superlattice (SL). ${ }^{3}$ Here, BO's are excited by the coherent superposition of several electronic Wannier-Stark (WS) states $^{4,5}$ with a common hole state by short laser pulses. The localization lengths of the electronic wave packets deviate from the semiclassical picture of BO's [Eq. (1)] if a fully quantum-mechanical calculation of the wave functions in the artificial semiconductor structure is used. ${ }^{6}$ Additionally, the amplitude of optically excited BO's depends on the exciting laser pulse width and energy that determine the initial conditions of the wave packets. ${ }^{7}$

Pathbreaking investigations on BO's have been performed in four-wave-mixing, ${ }^{8,9} \mathrm{THz}$ emission, ${ }^{10}$ and resonant transmittive electro-optic sampling (TEOS) experiments. ${ }^{11}$ In recent years, the experimental determination of the spatial amplitude of the oscillating electronic wave packets has become an objective of the studies. The amplitude determination of BO's in a SL has been reported in a $\mathrm{THz}$ emission experiment. $^{12}$ The amplitudes were calculated from the detected $\mathrm{THz}$ radiation power by applying a superradiance theory. Subsequently, the BO amplitude has been carefully determined in spectrally and time-resolved four-wave mixing experiments. ${ }^{13}$ Using this technique, the dependence of the amplitudes on the spectral position of the exciting laser pulses was investigated. ${ }^{14}$ Resonant TEOS experiments performed with optical detection spectrally integrated over WS transitions can only provide a qualitative analysis of the electronic dynamics due to nonlinearities of the electro-optic effect at the interband resonances.
In this paper, we present the off-resonant electro-optic detection of Bloch oscillations in a $\mathrm{GaAs} / \mathrm{Al}_{x} \mathrm{Ga}_{1-x} \mathrm{As}$ SL. BO's are excited resonantly, but detected electro-optically in the center of the band gap far away from optical transitions. This detection scheme enables the direct osbervation of the spatial carrier dynamics in amplitude and phase. The $\mathrm{BO}$ amplitudes are obtained in dependence on the applied electric fields and compared to the semiclassical localization lengths of BO's and a quantum-mechanical calculation of the oscillation amplitude of the electronic wave packets in the SL.

The investigated SL consists of 35 periods of $9.7 \mathrm{~nm}$ wide GaAs wells and $1.7 \mathrm{~nm}$ wide $\mathrm{Al}_{0.3} \mathrm{Ga}_{0.7} \mathrm{As}$ barriers. These are embedded between intrinsic $\mathrm{Al}_{0.3} \mathrm{Ga}_{0.7} \mathrm{As}$ buffer layers of $250 \mathrm{~nm}$ and $350 \mathrm{~nm}$ width resulting in an intrinsic region of $1 \mu \mathrm{m}$ which is electro-optically active. The structure is grown on an (001) oriented $n$-doped GaAs substrate and prepared as described in Ref. 11. The electronic miniband width is calculated to $18 \mathrm{meV}$ via a Kronig-Penney model. The narrow heavy-hole miniband $(1.9 \mathrm{meV})$ leads to a localization of heavy holes even at low electric fields.

The central wavelength of the laser pulses is chosen to excite electronic WS states and heavy holes. Since the heavy holes are assumed to be localized, the coherent and incoherent spatial dynamics of the electronic wave packets relative to the holes result in a dipole field $P_{z}(\tau, z)$ that leads to a voltage drop $\Delta V_{z}(\tau)=\int P_{z}(\tau, z) d z$ in the SL. In first approximation, the dipole field depends linearly on the relative displacement $s_{z}(\tau)$ between the distinct carriers and the longitudinal inhomogenous excitation density $n_{\text {exc }}(z)$ $\propto \exp (-\alpha z)$ by

$$
P(\tau, z)=\frac{e n_{e x c}(z)}{\epsilon \epsilon_{0}} s_{z}(\tau),
$$

where $\alpha$ is the absorption coefficient of the sample. These field dynamics can be detected via the electro-optic effect. In crystals of the $\overline{4} 2 m$ point group, like III-V semiconductor SL's, circularly polarized light propagating in the (001) growth direction becomes elliptically polarized due to the 


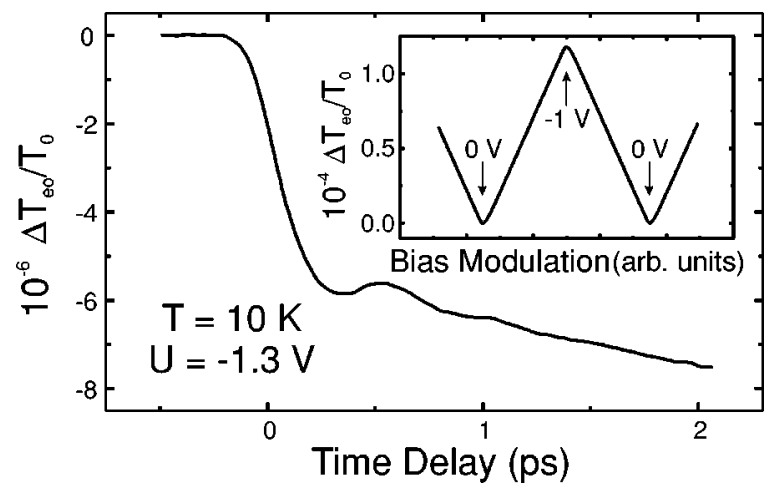

FIG. 1. Time-resolved TEOS signal at an excitation density of $2.7 \times 10^{9} \mathrm{~cm}^{-2}$ (in the first well) for an applied reverse bias of $-1.3 \mathrm{~V}$. The inset shows anisotropic transmission changes detected in a quasi-cw TEOS setup to determine the effective electro-optic coefficient of the investigated sample. The applied bias is modulated with a frequency of $4 \mathrm{kHz}$.

birefringence that is induced by a longitudinal field. The degree of the ellipticity can be detected by the subtraction of the transmitted beam intensities along the directions of the index ellipsoid's main axes (see, e.g., Refs. 11 and 15). The resulting TEOS signal depends linearly on the voltage drop $\Delta V_{z}$ over the electro-optic active length:

$$
\frac{\Delta T_{e o}}{T_{0}}=\frac{2 \pi}{\lambda} n_{0}^{3} r_{63} \Delta V_{z}=: A_{e o} \times \Delta V_{z} .
$$

Here, $T_{0}$ and $\lambda$ are the transmitted intensity and the wavelength of the probe beam, $n_{0}$ the refractice index, and $r_{63}$ the electro-optic coefficient of the crystal. $A_{e o}$ summarizes all voltage independent factors. Isotropic changes of the refractive index, e.g., due to free carrier absorption, ${ }^{16}$ do not contribute to the anisotropic TEOS signal.

The measurements are performed in a two-color pumpprobe setup at $10 \mathrm{~K}$ lattice temperature. The pump pulses of $125 \mathrm{fs}$ are derived from a Ti:sapphire laser at a central wavelength of $800 \mathrm{~nm}$. The laser beam is divided into one part for the excitation of the sample behind an external pulse compression and a second part to synchronously pump a lithium triborate (LBO)-based optical parametric oscillator (OPO). The probe pulses derived from the OPO are tuned to a central wavelength of $1.49 \mu \mathrm{m}$ with a length of $150 \mathrm{fs}$. Anisotropic transmission changes of the circularly polarized probe beam are detected behind a polarizing beamsplitter by two $\mathrm{In}_{x} \mathrm{Ga}_{1-x} \mathrm{As}$ photodiodes. The subtracted signal is sampled by a fast-scan data acquisition system (Aixscan, GWU Lasertechnik, Germany).

Due to the lack of literature values for electro-optic coefficients at $10 \mathrm{~K}$, it is incumbent to calibrate the anisotropic transmission changes to the voltage drop in the specific sample. Therefore, $A_{e o}$ [Eq. (3)] averaged over the spectral width of the probing light is measured in a quasi-cw (continous wave) TEOS experiment. Here, the anisotropic transmission changes are sampled phase-locked to the bias applied to the sample. The voltage is triangularly modulated from $0 \mathrm{~V}$ to $-1 \mathrm{~V}$ reverse bias at a frequency of $4 \mathrm{kHz}$. The detected transmission changes are shown in the inset of Fig. 1. The signal linearly follows the applied voltage with a slope equal the coefficient $A_{e o}=1.26 \times 10^{-4} \mathrm{~V}^{-1}$, which will be used for the quantitative analysis of the time-resolved experiments. From this value and an effective refractive index $n_{0}=3.20$ of the sample at $10 \mathrm{~K}$, the effective electrooptic coefficient is calculated to be $r_{63}^{*}=0.91 \times 10^{-12} \mathrm{~m} / \mathrm{V}$. This is in a good agreement with the room temperature value of $r_{63}=1.50 \times 10^{-12} \mathrm{~m} / \mathrm{V}$ at $1.52 \mu \mathrm{m}$ of bulk GaAs (Ref. 17) and the temperature dependence that has been investigated down to lattice temperatures of $100 \mathrm{~K}^{18} \mathrm{In}$ our calibration with a modulation frequency of $4 \mathrm{kHz}$, we measure the unclamped electro-optic coefficient. In the time-resolved experiments with $\mathrm{BO}$-induced electric-field modulations in the $\mathrm{THz}$ range, the clamped value has to be considered. With respect to room-temperature calculations of the clamped and unclamped values, we neglect the piezoelectrically induced lattice contributions to the electro-optic coefficient that are of opposite sign and assumed to be at least one order of magnitude smaller than those of the electronic subsystem. ${ }^{19}$

Figure 1 shows a time-resolved TEOS signal detected at a reverse bias of $-1.3 \mathrm{~V}$ and an excitation density of 2.7 $\times 10^{9} \mathrm{~cm}^{-2}$ (in the first well). The central wavelength of the pump beam is located between the WS $(-1)$ and the WS(0) transitions. Initially, the detected signal consists of a step that follows the integral over the laser intensity profile. This step is attributed to the electric-field induced instantaneous displacement of the electronic wave packet relative to the localized holes within the pulse duration that creates an initial dipole field. ${ }^{20}$ Additionally, the anisotropic transmission changes are periodically modulated due to the excitation of BO's. These data directly visualize the coherent excitation of BO's and the associated dipole field with a well defined phase relative to zero time delay. At excitation below the WS(0) transition, the electronic wave packets are initially displaced in the direction of the applied electric field. The cosinelike oscillation around this new "equilibrium" position starts in the same direction. This unidirectional movement of both components could not be extracted from $\mathrm{THz}$ emission, resonant TEOS, or four-wave mixing experiments that are not sensitive to the direction of the carrier motion at zero time delay. At larger time delays, the amplitude of the oscillation is damped and the negative background signal further increases. This effect results from the dephasing of the BOs that (i) reduces the strength of the associated dipole field due to the reduced number of coherently oscillating electrons and (ii) leads to a drift current of the scattered electrons through the SL which further screens the applied electric field. ${ }^{21}$ We should further note that the signals detected in resonant TEOS experiments are typically two orders of magnitude larger. ${ }^{11}$ This enlargement is attributed to the resonant enhancement of the electro-optic effect at the WS transitions.

For further analysis, anisotropic transmission changes are detected at reverse biases increasing from $-0.9 \mathrm{~V}$ to $-1.6 \mathrm{~V}$ in steps of $-0.1 \mathrm{~V}$, i.e., in the WS regime of the SL. By folding the exciting laser spectrum with the spectral absorption of the sample, the excitation density was calculated to vary less than $8 \%$ in the relevant voltage range caused by the electric-field dependence of the absorption strengths of WS transitions. This fluctuation is comparably weak to our experimental error in the determination of the excitation density. The oscillatory signal contributions extracted numerically are shown in Fig. 2. An increase of the 


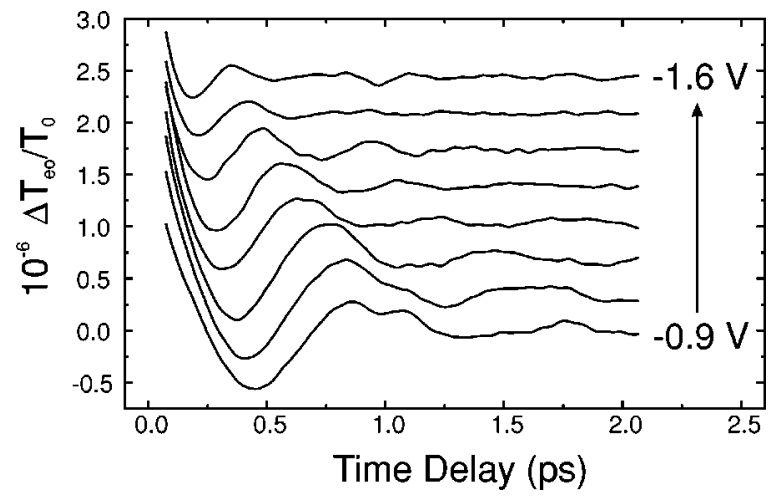

FIG. 2. Oscillatory contributions to the anisotropic transmission changes at reverse biases increasing from $-0.9 \mathrm{~V}$ to $-1.6 \mathrm{~V}$ in steps of -0.1 V. For clarity, the traces are shifted vertically.

oscillation frequency with increasing reverse bias is clearly visible manifesting the detection of BO's. Additionally, the amplitude of the oscillations decreases with increasing reverse biases. Since the excitation density is approximately constant for the applied electric fields, the decrease of the amplitude is caused by a reduction of the BO induced dipole field due to the enhanced localization at higher fields. The dephasing time of the detected BOs determined from monoexponentially decaying fits to the extracted oscillations increases from about $0.3 \mathrm{ps}$ at $-1.6 \mathrm{~V}$ to $0.5 \mathrm{ps}$ at $-0.9 \mathrm{~V}$. These values are below the longest dephasing times of 2.8 $\mathrm{ps},{ }^{11}$ but in the range of $0.37 \mathrm{ps},{ }^{22}$ both observed in resonant TEOS experiments at comparable excitation densities, but in different samples. This discrepancy is attributed to different sample qualitities, e.g., regarding the interface roughness, that strongly influence the coherence dephasing time.

The frequencies of the BO's obtained from Fourier transforms to the extracted oscillations are shown in the inset of Fig. 3. They increase linearly from $0.9 \mathrm{THz}$ to $2.7 \mathrm{THz}$ with increasing reverse biases. The slope obtained from a linear fit to the data is $2.5 \mathrm{THz} / \mathrm{V}$ which is in good agreement with the value expected from the given sample parameters by the semiclassical picture of BO's. This is calculated to $e d / h l_{e o}$ $=2.76 \mathrm{THz} / \mathrm{V}$, assuming that the applied voltage drops over the whole intrinsic region of the sample. The accumulation of carriers at the SL boundary layers between successive pump pulses leads to a quasistatic screening of the applied electric field. ${ }^{23}$ Its amount of $6 \mathrm{kV} / \mathrm{cm}$ is independent on the applied bias and thus in agreement with the assumption of a constant excitation density for different applied fields.

In the following, the oscillation amplitudes of the electronic wave packets are determined as a function of the applied electric field. Therefore, the extracted oscillations are extrapolated to zero time delay by numerical fits. From the signal amplitude at $\tau=0$, the spatial peak-to-peak amplitude of the oscillating electrons can be calculated considering the inhomogenous excitation density. The results are shown in Fig. 3 (squares) dependent on the electric fields that have been calculated from the peak positions of the Fourier transforms of the time-domain oscillations. The experimental errors mainly result from the determination of the excitation density. Error contributions due to the experimental equipment and the careful analysis of the time-domain data are of minor weight but not neglected. The obtained amplitudes

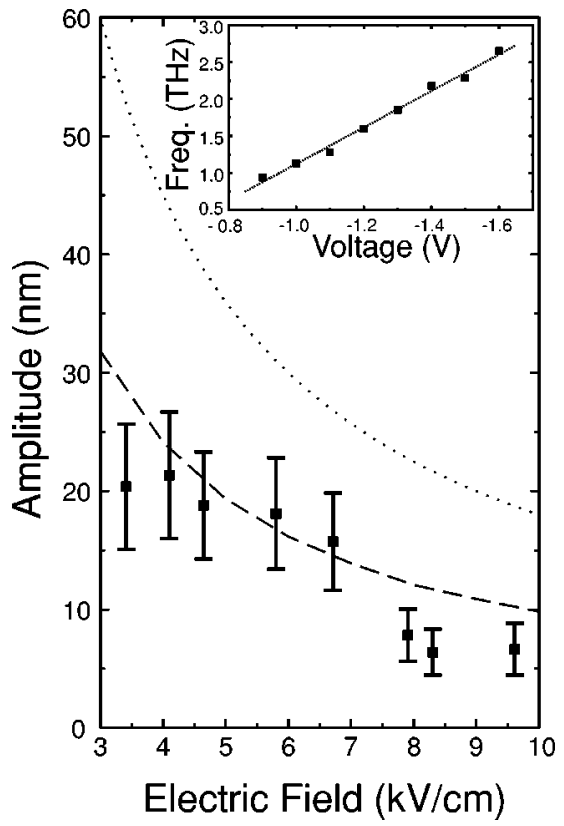

FIG. 3. Experimentally determined peak-to-peak amplitude of the oscillating electronic wave packets dependent on the electric fields (squares). The dashed and dotted lines are calculations from a quantum-mechanical and the semiclassical theory, respectively. The inset shows the peak positions of Fourier transforms to the Bloch oscillations and a linear fit to the data.

decrease from $21 \mathrm{~nm}$ at an electric field of $3.4 \mathrm{kV} / \mathrm{cm}$ to 6.5 $\mathrm{nm}$ at $9.6 \mathrm{kV} / \mathrm{cm}$. Thus, the $\mathrm{BO}$ amplitudes are in the range between less than one and two SL periods in this electricfield range. Obviously, the semiclassical BO amplitudes (dotted line) are about a factor of 2 larger than the experimentally detected amplitudes. This is in agreement with the results of Lyssenko et al. who quantified the oscillation amplitudes in a $38 \mathrm{meV}$ GaAs $/ \mathrm{Al}_{x} \mathrm{Ga}_{1-x}$ As superlattice. ${ }^{13}$ There, the wave packets reached about $2 / 3$ of the semiclassical amplitude. The discrepancy between experimentally determined and semiclassical amplitudes are assumed to result from the precise excitation conditions, i.e., the spectral laser position, and the deformation of the wave functions due to different effective masses in barriers and wells. Recently, it has been shown that the excitation of electronic wave packets near the WS(0) transition leads to a reduced center of mass motion due to the wave packet's distribution over a large part of the Brillouin zone with positive and negative masses. ${ }^{14}$ The associated breathing motion results in a reduced dipole field. Here, we compare the experimental results with a quantum-mechanical calculation of the electronic wave functions taking into account solely the difference of effective masses in the wells and barriers. ${ }^{6}$ The oscillation amplitudes are calculated in the assumption of the superposition of the WS(-1) and the WS(0) states by a modified Numerov algorithm (dashed line). ${ }^{23}$ A comparison between the calculated and the experimental data yields a good agreement within the experimental errors.

In conclusion, we realized a two-color pump-probe setup for quantitative investigations of electric-field dynamics in III-V semiconductors with femtosecond time resolution. The field dynamics are detected electro-optically in the center of the bandgap enabling the direct visualization of spatial car- 
rier dynamics after optical excitation. This new method complements the established time-resolved techniques of four-wave mixing, $\mathrm{THz}$ emission and resonant TEOS with the advantage that (i) the signal amplitudes can be calibrated, (ii) the detection is free from excitonic contributions, and the disadvantage of small signal amplitudes at the low excitation densities required for the observation of electronic coherence. Particularly, we monitored the space-time evolution of resonantly excited Bloch oscillations in a biased GaAs $/ \mathrm{Al}_{x} \mathrm{Ga}_{1-x} \mathrm{As}$ superlattice. The initial displacement, the coherent oscillations, and the incoherent transport of elec- tronic wave packets were clearly observed. The oscillation amplitudes of the electronic wave packets increase from less than one to about two superlattice periods in the investigated electric field range. The results can be modeled by quantummechanical calculations of the oscillation amplitudes in the superlattice.

The authors gratefully acknowledge G.C. Cho for stimulating suggestions and R. Martini for helpful discussions. This work was supported by the Volkswagen Stiftung.
*Electronic address: foerst@iht-ii.rwth-aachen.de

${ }^{1}$ F. Bloch, Z. Phys. 52, 555 (1928).

${ }^{2}$ C. Zener, Proc. R. Soc. London, Ser. A 145, 523 (1932).

${ }^{3}$ L. Esaki and R. Tsu, IBM J. Res. Dev. 61, 61 (1970).

${ }^{4}$ E. E. Mendez, F. Agullo-Rueda, and J. M. Hong, Phys. Rev. Lett. 60, 2426 (1988).

${ }^{5}$ P. Voisin, J. Bleuse, C. Bouche, S. Gaillard, C. Alibert, and A. Regreny, Phys. Rev. Lett. 61, 1639 (1988).

${ }^{6}$ See, e.g., F.T. Vasko and A.V. Kuznetsov, Electronic States and Optical Transitions in Semiconductor Heterostructures (Springer, New York, 1999), p. 80.

${ }^{7}$ M. M. Dignam, J. E. Sipe, and J. Shah, Phys. Rev. B 49, 10502 (1994).

${ }^{8}$ J. Feldmann, K. Leo, J. Shah, D. A. B. Miller, J. E. Cunningham, S. Schmitt-Rink, T. Meier, G. von Plessen, A. Schulze, and P. Thomas, Phys. Rev. B 46, 7252 (1992).

${ }^{9}$ K. Leo, P. Haring-Bolivar, F. Brüggemann, R. Schwedler, and K. Köhler, Solid State Commun. 84, 943 (1992).

${ }^{10}$ C. Waschke, H. G. Roskos, R. Schwedler, K. Leo, H. Kurz, and K. Köhler, Phys. Rev. Lett. 70, 3319 (1993).

${ }^{11}$ T. Dekorsy, P. Leisching, K. Köhler, and H. Kurz, Phys. Rev. B 50, 8106 (1994).

${ }^{12}$ R. Martini, G. Klose, H. G. Roskos, H. Kurz, H. T. Grahn, and R.
Hey, Phys. Rev. B 54, 14325 (1996).

${ }^{13}$ V. G. Lyssenko, G. Valušis, F. Löser, T. Hasche, K. Leo, M. M. Dignam, and K. Köhler, Phys. Rev. Lett. 79, 301 (1997).

${ }^{14}$ M. Sudzius, V. G. Lyssenko, F. Löser, K. Leo, M. M. Dignam, and K. Köhler, Phys. Rev. B 57, R12 693 (1998).

${ }^{15}$ S. E. Ralph, F. Capasso, and R. J. Malik, Phys. Rev. Lett. 63, 2272 (1989).

${ }^{16}$ J. Faist and F. K. Reinhart, J. Appl. Phys. 67, 6998 (1990).

${ }^{17}$ C. A. Berseth, C. Wuethrich, and F. K. Reinhart, J. Appl. Phys. 71, 2821 (1992).

${ }^{18}$ Y. V. Shaldin and D. A. Belogurov, Sov. J. Quantum Electron. 6, 897 (1976).

${ }^{19}$ S. Adachi and K. Oe, J. Appl. Phys. 56, 74 (1984).

${ }^{20}$ H. G. Roskos, M. C. Nuss, J. Shah, K. Leo, D. A. B. Miller, A. M. Fox, S. Schmitt-Rink, and K. Köhler, Phys. Rev. Lett. 68, 2216 (1992).

${ }^{21}$ M. Först, G. C. Cho, T. Dekorsy, and H. Kurz, Superlattices Microstruct. 26, 83 (1999).

${ }^{22}$ T. Dekorsy, R. Ott, H. Kurz, and K. Köhler, Phys. Rev. B 51, 17 275 (1995).

${ }^{23}$ P. Leisching, P. Haring-Bolivar, W. Beck, Y. Dhaibi, F. Brüggemann, R. Schwedler, H. Kurz, K. Leo, and K. Köhler, Phys. Rev. B 50, 14389 (1994). 